\title{
Electronic prompt for capillary blood glucose monitoring in patients admitted for a flare of inflammatory bowel disease increases detection of steroid-induced diabetes
}

\author{
Authors: Benjamin Mitchell, Ben Zare, Tilly Steane, Yathevan Abayalingam and Markus Gwiggner
}

\begin{abstract}
Aims
To improve monitoring of capillary blood sugars (CBG) and diagnosis of steroid induced diabetes in patients receiving high dose steroids to treat a flare of inflammatory bowel disease (IBD).

\section{Methods}

Frequency of CBG monitoring for patients admitted with a flare of IBD without a prior diagnosis of diabetes between February-April 2017 was recorded. Following the introduction of an electronic recording system for $\mathrm{CBG}$ a re-audit took place for patients admitted between June-August 2017. Our second intervention introduced an electronic prompt for the nursing staff reminding them to monitor CBG while they charted steroid administration. Following this, a prospective database was collected monitoring from December 2017 to January 2018. Frequency of monitoring was compared to the recommended frequency, by Joint British Diabetes Societies for Inpatient Care (JBDS-IC), of once daily postprandial levels. Blood sugars were recorded over the first 4 days of starting steroid therapy.
\end{abstract}

\section{Results}

Nine patients were identified for inclusion between FebruaryApril 2017. Zero to $11 \%$ of patients received once daily CBGs on day one-four of admission. Sixteen patients were identified for inclusion between June-August 2017. With electronic recording of CBGs the percentage of patients with a minimum of once daily CBG monitoring increased. Twenty-one patients were identified for inclusion between December 2017 and January 2018. Following the introduction of an electronic prompt, the percentage of patients receiving once daily CBGs increased to $71-95 \%$. In this prospective dataset, $60 \%$ of patients were found to have steroid induced diabetes.

\section{Conclusion}

Introduction of an electronic prompt to check CBGs once daily for patients receiving high dose steroids greatly increases the percentage of patients with adequate CBG monitoring as recommended by JBDS-IC. Monitoring of CBGs is essential for the diagnosis of steroid induced diabetes. In our dataset, $60 \%$ of patients admitted would not have been found to have a diagnosis of steroid induced diabetes without once daily CBG monitoring. Further work is needed to establish the risk factors for steroid induced diabetes in IBD and as to whether treatment of this condition improves medical and surgical outcomes for these patients.

\section{Conflict of interest statement}

None declared. 